Pacific

Journal of

Mathematics

\title{
ASYMPTOTIC LIFTS OF POSITIVE LINEAR MAPS
}

William ARVESON AND ERLING StøRmER 


\title{
ASYMPTOTIC LIFTS OF POSITIVE LINEAR MAPS
}

\author{
William ARVESON AND ERLING StøRMER
}

\begin{abstract}
We show that the notion of asymptotic lift generalizes naturally to normal positive maps $\phi: M \rightarrow M$ acting on von Neumann algebras $M$. We focus on cases in which the domain of the asymptotic lift can be embedded as an operator subsystem $M_{\infty} \subseteq M$ and characterize when $M_{\infty}$ is a Jordan subalgebra of $M$ in terms of the asymptotic multiplicative properties of $\phi$.
\end{abstract}

\section{Introduction}

Let $\phi: M \rightarrow M$ be a normal unit-preserving positive linear map acting on a dual operator system $M$; we refer to such a pair $(M, \phi)$ as a $U P$ map. While we are primarily interested in UP maps that act on von Neumann algebras $M$, it is useful to broaden the context as above. The powers of $\phi$ form an irreversible dynamical semigroup $\left\{\phi^{n}: n=0,1,2, \ldots\right\}$ acting on $M$. In this paper we generalize work begun in [Arveson 2004; 2007], together with complementary results in [Størmer 2007], to further develop the asymptotic theory of such semigroups.

One may view UP maps as the objects of a category, in which a homomorphism from $\phi_{1}: M_{1} \rightarrow M_{1}$ to $\phi_{2}: M_{2} \rightarrow M_{2}$ is a UP map $E: M_{1} \rightarrow M_{2}$ such that $E \circ \phi_{1}=\phi_{2} \circ E$. There is a natural notion of isomorphism in this category. Our first general result is that every normal unit-preserving positive linear map acting on a dual operator system has an asymptotic lift which is unique up to isomorphism. This generalizes one of the main results of [Arveson 2007], which dealt with the subcategory in which the objects are unital normal completely positive maps (UCP maps) on dual operator systems, with UCP maps as morphisms.

We are primarily concerned with UP maps that act on von Neumann algebras $M$. In [Arveson 2007], it was shown that the asymptotic lift $(N, \alpha, E)$ of a UCP map $\phi: M \rightarrow M$ acting on a von Neumann algebra $M$ also acts on a von Neumann algebra $N$. Moreover, it was shown that the $W^{*}$-dynamical system $(N, \alpha)$ can be identified with the tail flow of the minimal dilation of $\phi$ to a $*$-endomorphism of a larger von Neumann algebra in most cases - namely, those in which the dilation endomorphism has trivial kernel, which includes all UCP maps acting on factors $M$. Since the minimal dilation of a UCP map on a von Neumann algebra can be

MSC2000: primary 46L55; secondary 46L40.

Keywords: von Neumann algebras, positive maps, ergodic theory, asymptotic lifts. 
constructed explicitly in principle, that provided a concrete identification of the asymptotic lift.

It is significant that the asymptotic lift $(N, \alpha, E)$ of a UP map acting on a von Neumann algebra need not act on a von Neumann algebra $N$. In this paper we show that, in general, $N$ is order-isomorphic to a unique $\mathrm{JW}^{*}$-algebra - namely, a dual operator system that is closed under the Jordan product $x \circ y=(x y+y x) / 2-$ so that $\alpha$ becomes a Jordan automorphism of $N$. Thus, the asymptotic behavior of a UP map on a von Neumann algebra is always associated with a $\mathrm{JW}^{*}$-dynamical system $(N, \alpha)$.

Naturally, one would like to identify $(N, \alpha)$ more concretely in terms of the asymptotic properties of the semigroup $\phi, \phi^{2}, \phi^{3}, \ldots$ But since there is no dilation theory for semigroups of UP maps - and perhaps an effective dilation theory is impossible - there seems to be no candidate to replace the "tail flow" identification described above for UCP maps. Thus, this identification problem becomes a significant issue for UP maps.

In many cases, $N$ can be embedded as an operator system $N \subseteq M$ so that $\alpha=$ $\phi \uparrow_{N}$. While the operator system $N$ is order-isomorphic to a $\mathrm{JW}^{*}$-algebra in general, it need not be closed under the ambient Jordan multiplication of $M$, and we address that issue in Section 4. We identify $N$ concretely in terms of the action of $\phi$ on $M$, and when $N$ is a Jordan subalgebra of $M$, we are able to go farther by identifying $N$ with the multiplicative core of $\phi$ introduced in [Størmer 2007].

The problem of finding a satisfactory concrete description of the $\mathrm{JW}^{*}$-dynamical system $(N, \alpha)$ when $N$ does not embed in $M$ remains untouched.

\section{Asymptotic lifts of positive maps}

In this section we describe how the notion of asymptotic lift (of a completely positive map) can be generalized to normal unit-preserving maps that are merely positive. We summarize the basic properties of asymptotic lifts, indicating briefly how proofs of [Arveson 2007] should be modified.

Remark 2.1 (Dual operator systems, norm, and order). Every von Neumann algebra is the dual of a unique Banach space, and hence it carries a natural weak* topology (also known as the ultraweak or $\sigma$-weak topology). A dual operator system is a linear space of operators $M \subseteq \mathscr{B}(H)$ that contains the identity $\mathbf{1}$, is self-adjoint $M^{*}=M$, and is closed in the weak*-topology of $\mathscr{B}(H)$. Such an $M$ also has a unique predual $M_{*}$, and its intrinsic $M_{*}$-topology coincides with the relative weak*-topology of $\mathscr{B}(H)$. A map of dual operator systems $\phi: M \rightarrow N$ is called normal when it is weak*-continuous. There is an intrinsic characterization of dual operator systems, which we do not require. 
Let us recall the basic properties of unit-preserving normal positive linear maps $\phi: M \rightarrow N$ (UP maps) between dual operator systems. A UP map $\phi: M \rightarrow N$ defined on a $C^{*}$-algebra $M$ must satisfy $\|\phi\|=1$. That is most easily seen by making use of the Russo-Dye theorem [1966] which implies that $\|\phi\|=\sup \|\phi(u)\|$, where $u$ ranges over the unitary group of $M$, together with the fact that for every unitary $u$, the restriction of $\phi$ to the commutative $C^{*}$-algebra $C^{*}(u)$ is completely positive and therefore satisfies the strong Schwarz inequality $\phi(u)^{*} \phi(u) \leq \phi\left(u^{*} u\right)=\phi\left(\mathbf{1}_{M}\right)=$ $\mathbf{1}_{N}$. More generally, if $M$ is merely an operator system, then one has $1 \leq\|\phi\| \leq 2$ in general, but the $C^{*}$-algebraic upper bound $\|\phi\|=1$ often fails.

On the other hand, since the norm of a self-adjoint operator $x$ is the smallest $\alpha \geq 0$ such that $-\alpha \mathbf{1} \leq x \leq \alpha \mathbf{1}$, the restriction of $\phi$ to the real Banach space $M^{\text {sa }}$ of self-adjoint elements of $M$ has norm 1. Conversely, if a linear map $\phi$ : $M \rightarrow N$ carries self-adjoint elements to self-adjoint elements, maps $\mathbf{1}_{M}$ to $\mathbf{1}_{N}$, and satisfies $\| \phi\left\lceil_{M^{\text {sa }}} \|=1\right.$, then $\phi$ must also preserve positivity. In particular, an order isomorphism in the category of UP maps is characterized as a $*$-preserving linear map $\phi: M \rightarrow N$ of operator systems that carries $\mathbf{1}_{M}$ to $\mathbf{1}_{N}$ and restricts to an isometry of $M^{\mathrm{sa}}$ onto $N^{\mathrm{sa}}$.

Definition 2.2. A reversible lift of a UP map $\phi: M \rightarrow M$ is a triple $(N, \alpha, E)$ consisting of a UP automorphism $\alpha: N \rightarrow N$ of another dual operator system $N$ and a UP map $E: N \rightarrow M$ satisfying $E \circ \alpha=\phi \circ E$.

A reversible lift $(N, \alpha, E)$ of $\phi$ is said to be nondegenerate if

$$
E\left(\alpha^{-n}(y)\right)=0 \text { for } n=0,1,2, \ldots \quad \text { implies } y=0 .
$$

Significantly, if (2-1) fails, one can replace $(N, \alpha, E)$ with another reversible lift $(\tilde{N}, \tilde{\alpha}, \tilde{E})$ that is nondegenerate, as in [Arveson 2007, Remark 2.3].

Definition 2.3. Let $\phi: M \rightarrow M$ be a UP map on a dual operator system. An asymptotic lift of $\phi$ is a reversible lift $(N, \alpha, E)$ of $\phi$ that satisfies nondegeneracy (2-1) together with

$$
\left\|\rho \circ E \uparrow_{N^{\text {sa }}}\right\|=\lim _{k \rightarrow \infty} \| \rho \circ \phi^{k}\left\lceil_{M^{\text {sa }}} \| \text { for } \rho \in M_{*} .\right.
$$

Remark 2.4. We shall make use of the dual formulation of (2-2), and we record that now for later reference: For every nondegenerate reversible lift $(N, \alpha, E)$ of $\phi,(2-2)$ is equivalent to the assertion

$$
E\left(\operatorname{ball}_{r} N^{\mathrm{sa}}\right)=\bigcap_{n=1}^{\infty} \phi^{n}\left(\operatorname{ball}_{r} M^{\mathrm{sa}}\right) \quad \text { for } r>0,
$$

where ball ${ }_{r} X$ denotes the closed ball of radius $r$ in a real or complex Banach space $X$. The proof that (2-2) and (2-3) are equivalent follows along the lines of the proof of the corresponding result in [Arveson 2007]. 
There are two fundamental results on asymptotic lifts of UP maps. The first concerns existence and uniqueness:

Theorem 2.5. Every UP map $\phi: M \rightarrow M$ of a dual operator system has an asymptotic lift. If $\left(N_{1}, \alpha_{1}, E_{1}\right)$ and $\left(N_{2}, \alpha_{2}, E_{2}\right)$ are two asymptotic lifts for $\phi$, then there is a unique UP-isomorphism of dual operator systems $\theta: N_{1} \rightarrow N_{2}$ such that $\theta \circ \alpha_{1}=\alpha_{2} \circ \theta$ and $E_{2} \circ \theta=E_{1}$.

As in the case of completely positive maps, the existence issue is settled by a direct construction based on inverse sequences, namely bounded bilateral sequences $\left(x_{n}\right)$ of elements of $M$ that satisfy $x_{n}=\phi\left(x_{n+1}\right)$ for $n \in \mathbb{Z}$. The space of all inverse sequences is a dual operator system $N$, the bilateral shift $\alpha:\left(x_{n}\right) \mapsto\left(x_{n-1}\right)$ is an automorphism of $N$, and the connecting map $E$ carries $\left(x_{n}\right)$ to $x_{0}$. The proof that $(N, \alpha, E)$ is an asymptotic lift is a minor (and somewhat simpler) variation of the proof of the corresponding result in [Arveson 2007], and, similarly, the proof of uniqueness is a straightforward variation of arguments therein; we omit the details.

If a UP map $\phi: M \rightarrow M$ on a von Neumann algebra $M$ is completely positive, then its asymptotic lift $(N, \alpha, E)$ gives rise to a $W^{*}$-dynamical system $(N, \alpha)$ [Arveson 2007]. While this need not be true for asymptotic lifts of UP maps, one can assert in general:

Theorem 2.6. Let $\phi: M \rightarrow M$ be a UP map on a von Neumann algebra $M$ and let $(N, \alpha, E)$ be its asymptotic lift. Then $N$ is order isomorphic to a unique JW*algebra such that $\alpha$ is a Jordan automorphism of $N$.

Again, the proof follows along the lines of arguments in [Arveson 2007], by introducing a Jordan multiplication on the range of a positive idempotent map as in [Effros and Størmer 1979, Corollary 1.6]. The key step shows that for the constructed asymptotic lift $(N, \alpha, E)$ in which $N$ is the space of inverse sequences, there is a projection $Q: \ell^{\infty}(M) \rightarrow N$ of norm one. The existence of such a projection follows from the argument of [Arveson 2007]. Once one has a positive projection (which in the current setting is typically not completely positive) with these properties, one can introduce a Jordan product in $N$ by way of

$$
x \circ y=Q\left(\frac{1}{2}(x y+y x)\right) \text { for } x, y \in N,
$$

and at this point one can show that $N$ is order isomorphic to a $\mathrm{JW}^{*}$-algebra by imitating arguments in [Arveson 2007].

\section{Embeddable asymptotic lifts}

Let $(N, \alpha, E)$ be the asymptotic lift of a UP map $\phi: M \rightarrow M$ acting on a dual operator system. In this section we fix attention on those cases in which the asymptotic lift can be embedded as a subsystem of $M$ in the following particular way. We say 
that $(N, \alpha, E)$ embeds in $M$ if $E$ is restricts to an isometry on the self-adjoint part of $N$ :

$$
\|E(y)\|=\|y\| \quad \text { for } y=y^{*} \in N .
$$

This is equivalent to the claim that $E$ implements an order isomorphism of $N$ onto $E(N)$. Since all asymptotic lifts of $\phi: M \rightarrow M$ are isomorphic, this definition does not depend on the particular choice of $(N, \alpha, E)$. In such cases, the range $E(N)$ of $E$ is an operator subsystem of $M$ with the property that $\phi$ restricts to an automorphism of $E(N), \alpha$ is identified with $\phi \uparrow_{E(N)}$, and $E$ is identified with the inclusion map $\iota: E(N) \subseteq M$.

The purpose of this section is to precisely summarize those facts and, especially, to explicitly describe $E(N)$ in terms of the action of $\phi$ on $M$. We begin by introducing an operator system $M_{\infty} \subseteq M$ :

$$
M_{\infty}=\bigcup_{r>0} \bigcap_{n=1}^{\infty} \phi^{n}\left(\operatorname{ball}_{r} M\right)
$$

$M_{\infty}$ consists of all $y \in M$ for which there is a bounded sequence $x_{n} \in M$ with $y=\phi^{n}\left(x_{n}\right)$ for $n=1,2, \ldots$ In general, $M_{\infty}$ is a self-adjoint linear subspace of $M$ containing the identity operator, it is invariant under $\phi$, and in fact $\phi\left(M_{\infty}\right)=M_{\infty}$. Moreover, from (2-3) we may infer that

$$
E\left(N^{\mathrm{sa}}\right)=\bigcup_{r>0} E\left(\operatorname{ball}_{r} N^{\mathrm{sa}}\right)=\bigcup_{r>0} \bigcap_{n=1}^{\infty} \phi^{n}\left(\operatorname{ball}_{r} M^{\mathrm{sa}}\right)=M_{\infty}^{\mathrm{sa}} ;
$$

hence $M_{\infty}=E(N)$ is precisely the range of $E$ in all cases. We sometimes refer to $M_{\infty}$ as the tail operator system of $M$.

Remark 3.1. It is clear that $M_{\infty} \subseteq \cap_{n} \phi^{n}(M)$, but the inclusion is typically proper. As a simple example, let $M=M_{2}(\mathbb{C})$ and consider the completely positive map $\phi: M \rightarrow M$ defined by

$$
\phi\left(\begin{array}{ll}
a & b \\
c & d
\end{array}\right)=\left(\begin{array}{cc}
a & \lambda b \\
\lambda c & d
\end{array}\right)
$$

where $\lambda$ is a constant satisfying $0<\lambda<1$. One has $\cap_{n} \phi^{n}(M)=M$, but in this case, $M_{\infty}$ is the two-dimensional subalgebra of diagonal matrices.

Proposition 3.2. Let $(N, \alpha, E)$ be an asymptotic lift of $\phi: M \rightarrow M$. Then $\operatorname{ker} E=$ $\{0\}$ if and only if the restriction of $\phi$ to $M_{\infty}$ is both injective and surjective.

Proof. In general, one has $\phi\left(M_{\infty}\right)=M_{\infty}$ by definition of $M_{\infty}$. Assuming that $\operatorname{ker} E=\{0\}$, choose $a \in M_{\infty}$ such that $\phi(a)=0$. Since $E(N)=M_{\infty}$, there is a $y \in N$ such that $a=E(y)$; hence $0=\phi(a)=\phi(E(y))=E(\alpha(y))$, and therefore 
$\alpha(y)=0$ because $E$ is injective. $y=0$ follows because $\alpha$ is an automorphism of $N$; hence $a=E(y)=0$.

Conversely, if the restriction of $\phi$ to $M_{\infty}$ is injective, choose a nonzero element $y \in N$. Since the norms $\left\|E\left(\alpha^{-n}(y)\right)\right\|$ increase to $\|y\|$ as $n \uparrow \infty$ (see [Arveson 2007, Lemma 3.7]), we must have $E\left(\alpha^{-n}(y)\right) \neq 0$ for sufficiently large $n \geq 1$. Since each power $\phi^{n}$ restricts to an injective map on $M_{\infty}=E(N)$, it follows that

$$
E(y)=\phi^{n}\left(E\left(\alpha^{-n}(y)\right)\right) \neq 0
$$

for large $n$; hence $E$ is injective.

We conclude that whenever ker $E=\{0\}$, the restriction of $\phi$ to $M_{\infty}$ defines an order-preserving linear bijection on $M_{\infty}$. However, $M_{\infty}$ itself need not be closed in any topology in general, and the restriction of $\phi$ to $M_{\infty}$ need not be an order automorphism. The following result implies that when $E$ restricts to an isometry on $N^{\mathrm{sa}}$, such anomalies cannot occur.

Theorem 3.3. For every UP map $\phi: M \rightarrow M$ on a dual operator system, these are equivalent:

(i) The asymptotic lift of $\phi: M \rightarrow M$ embeds in $M$.

(ii) $M_{\infty}$ is weak ${ }^{*}$-closed, and $\phi$ restricts to an order automorphism of it.

(iii) The asymptotic lift of $\phi: M \rightarrow M$ is isomorphic to the triple $\left(M_{\infty}, \phi \uparrow_{\infty}, \iota\right)$, where $\iota: M_{\infty} \subseteq M$ is the inclusion map.

Proof. Let $(N, \alpha, E)$ be an asymptotic lift of $\phi: M \rightarrow M$.

(i) $\Longrightarrow$ (ii): By hypothesis, $E$ restricts to an isometry of $N^{\text {sa }}$ onto the selfadjoint part of $E(N)=M_{\infty}$. Since $M_{\infty}$ is a self-adjoint linear space of operators and the adjoint operation is weak*-continuous, we will know $M_{\infty}$ is weak ${ }^{*}$-closed if we can show that its self-adjoint part $M_{\infty}^{\text {sa }}$ is weak*-closed. By a standard result on the weak*-closure of convex sets in dual Banach spaces, this will follow if we show that for every $r>0$, the intersection $M_{\infty}^{\mathrm{sa}} \cap$ ball $_{r} M$ of $M_{\infty}^{\mathrm{sa}}$ with the $r$-ball of $M$ is weak*-closed. Now the connecting map $E: N \rightarrow M$ restricts to a weak* ${ }^{*}$-continuous isometry of $N^{\text {sa }}$ onto $M_{\infty}^{\text {sa }}$, and by (2-3), it carries ball $N^{\text {sa }}$ onto $M_{\infty}^{\mathrm{sa}} \cap$ ball $_{r} M$. Since the unit ball of $N$ is weak*-compact, it follows that $M_{\infty}^{\text {sa }} \cap$ ball $_{r} M=E\left(\right.$ ball $\left._{r} N^{\mathrm{sa}}\right)$ is weak*-compact and hence weak*-closed. We conclude that $M_{\infty}^{\text {sa }}$ is weak*-closed.

Making use of Proposition 3.2, let $\psi$ be the linear automorphism of $M_{\infty}$ inverse to $\phi \uparrow_{M_{\infty}}$. Then since $E \circ \alpha=\phi \circ E$, we have $E \circ \alpha^{-1}=\psi \circ E$. Since both $E$ and $\alpha^{-1}$ restrict to isometries on $N^{\text {sa }}$, it follows that $\psi$ restricts to an isometry on $M_{\infty}^{\mathrm{sa}}=E\left(N^{\mathrm{sa}}\right)$. Since $\psi(\mathbf{1})=\mathbf{1}, \psi$ must also be order preserving, and we conclude that $\phi \uparrow_{M_{\infty}}$ is an order automorphism. 
(ii) $\Longrightarrow$ (iii): Under the hypothesis (ii), $\left(M_{\infty}, \phi \uparrow_{M_{\infty}}, \iota\right)$ becomes a reversible lift of $\phi: M \rightarrow M$ which is obviously nondegenerate. We show that it is the asymptotic lift of $\phi: M \rightarrow M$ by establishing (2-2). For that, consider the decreasing sequence of weak*-compact sets $\phi^{n}$ (ball $M^{s a}$ ) for $n=1,2, \ldots$, and choose a normal linear functional $\rho \in M_{*}$. By (2-3), we have

$$
\bigcap_{n=1}^{\infty} \phi^{n}\left(\text { ball } M^{s a}\right)=E\left(\text { ball } N^{\mathrm{sa}}\right) .
$$

Since $\rho$ is weak*-continuous, it follows from Lemma 3.5 of [Arveson 2007]) that

$$
\begin{aligned}
\left\|\rho \circ \phi^{n} \uparrow_{M_{\infty}}^{\mathrm{sa}}\right\| & =\sup \left\{\rho(x): x \in \phi^{n}\left(\text { ball } M^{\mathrm{sa}}\right)\right\} \downarrow \sup \left\{\rho(x): x \in E\left(\text { ball } N^{\mathrm{sa}}\right)\right\} \\
& =\left\|\rho \circ E \uparrow_{N^{\mathrm{sa}}}\right\|
\end{aligned}
$$

as $n \uparrow \infty$, and (iii) follows.

The implication (iii) $\Longrightarrow$ (i) is obvious.

Taken together, Theorems 3.3 and 2.6 imply:

Corollary 3.4. Let $\phi: M \rightarrow M$ be a UP map on a von Neumann algebra $M$ whose asymptotic lift embeds in $M$. Then the tail operator system $M_{\infty}$ is orderisomorphic to a JW*-algebra such that the restriction of $\phi$ to $M_{\infty}$ becomes a Jordan automorphism, and the asymptotic lift of $\phi$ is the triple $\left(M_{\infty}, \phi\left\lceil M_{\infty}, \iota\right)\right.$, where $\iota$ is the inclusion map $\iota: M_{\infty} \subseteq M$.

The asymptotic lifts of many UP maps are embeddable. That is true for all examples covered by the hypotheses of [Arveson 2004], and in Section 5 below, we elaborate on the special case of maps on finite-dimensional algebras. Not all infinite-dimensional UP maps are embeddable, as the next example illustrates.

Example 3.5. Let $L^{2}=L^{2}(\mathbb{T}, d \theta /(2 \pi))$, let $H^{2} \subseteq L^{2}$ be the usual Hardy space, and let $s \in \mathscr{B}\left(H^{2}\right)$ be the unilateral shift. Consider the UCP map $\phi$ defined on $M=\Re\left(H^{2}\right)$ by

$$
\phi(x)=s^{*} x s \text { for } x \in \mathscr{B}\left(H^{2}\right) .
$$

Let $u \in \mathscr{B}\left(L^{2}\right)$ be the bilateral shift and let $p$ be the projection of $L^{2}$ on $H^{2}$. Then the asymptotic lift of $\phi$ is the triple $\left(\mathscr{B}\left(L^{2}\right), \alpha, E\right)$, where $\alpha(y)=u^{*} y u$, and $E$ is the compression map $E(y)=p y \uparrow_{H^{2}}$. Since $\operatorname{ker} E \neq\{0\}$, the asymptotic lift of $\phi$ is not embeddable. We omit the calculations.

\section{Embedding and the multiplicative core}

If the asymptotic lift of a UP map $\phi: M \rightarrow M$ acting on a von Neumann algebra embeds in $M$, then by Corollary $3.4, M_{\infty}$ is order isomorphic to a $\mathrm{JW}^{*}$-algebra, and the restriction of $\phi$ to $M_{\infty}$ becomes a Jordan automorphism of $M_{\infty}$. Notice 
that this does not imply that $M_{\infty}$ itself is closed under the Jordan product $x \circ y=$ $(x y+y x) / 2$ inherited from $M$. Section 5 contains a simple example that illustrates the phenomenon.

In the present section, we study the Jordan structure of $M_{\infty}$ in more detail for the case when the asymptotic lift embeds in $M$. In particular, we show that if $M_{\infty}$ is itself a JW*-algebra, then it coincides with the multiplicative core $C_{\phi}$ of $\phi$ introduced in [Størmer 2007]. We also give necessary and sufficient conditions for $\left(C_{\phi}, \phi\left\lceil_{C_{\phi}}, \iota\right)\right.$ to be the asymptotic lift.

Following Størmer, the definite set of a UP map $\phi: M \rightarrow M$ is defined by

$$
M_{\phi}=\left\{x \in M: \phi\left(x^{*} \circ x\right)=\phi(x)^{*} \circ \phi(x)\right\} ;
$$

it is a $\mathrm{JW}^{*}$-algebra, and we have

$$
\phi(x \circ y)=\phi(x) \circ \phi(y) \quad \text { for } x \in M_{\phi} \text { and } y \in M .
$$

Continuing to follow Størmer, one can show that

$$
B_{\phi}=\left\{x \in M: \phi^{n}(x) \in M_{\phi}, \quad n=0,1,2, \ldots\right\}
$$

is a $\mathrm{JW}^{*}$-algebra on which $\phi$ restricts to a Jordan endomorphism, and one forces surjectivity on $\phi$ by restricting it to the "tail" subalgebra

$$
C_{\phi}=\bigcap_{n=1}^{\infty} \phi^{n}\left(B_{\phi}\right) .
$$

$C_{\phi}$ is called the multiplicative core of $\phi$. We require the following variation of [Størmer 2007, Lemma 6].

Lemma 4.1. The multiplicative core is the largest $J W^{*}$-subalgebra $N \subseteq M$ with

(i) $\phi(N)=N$, and

(ii) $\phi(x \circ y)=\phi(x) \circ \phi(y)$ for all $x, y \in N$.

Proof. Let $N$ be a $\mathrm{JW}^{*}$-subalgebra of $M$ with properties (i) and (ii), and choose $x \in N$. Then we have

$$
\phi\left(\phi^{n}(x)^{*} \circ \phi^{n}(x)\right)=\phi^{n+1}(x)^{*} \circ \phi^{n+1}(x) \text { for } n \geq 0,
$$

because $\phi$ is a Jordan endomorphism of $N$; hence $N \subseteq B_{\phi}$. It follows that $N=$ $\phi^{n}(N) \subseteq \phi^{n}\left(B_{\phi}\right)$ for every $n \geq 0$, so that $N \subseteq \cap_{n} \phi^{n}\left(B_{\phi}\right)=C_{\phi}$.

The following result associates the multiplicative core with asymptotic lifts that are embeddable as Jordan subalgebras:

Theorem 4.2. For every UP map $\phi: M \rightarrow M$ acting on a von Neumann algebra $M$, the tail operator system $M_{\infty}$ contains the multiplicative core $C_{\phi}$. 
Assume that the asymptotic lift embeds in $M$. Then $M_{\infty}$ is a Jordan subalgebra of $M$ precisely when $M_{\infty}=C_{\phi}$.

Proof. By definition, $C_{\phi}=\cap_{n} \phi^{n}\left(B_{\phi}\right)$, and $\phi$ restricts to a Jordan endomorphism of $B_{\phi}$. Since each power $\phi^{n}$ of $\phi$ restricts to a Jordan endomorphism on $B_{\phi}$ and a Jordan homomorphism maps the unit ball of the self-adjoint part of its domain onto the unit ball of the self-adjoint part of its range, we have $\phi^{n}\left(\operatorname{ball}_{r} B_{\phi}^{\mathrm{sa}}\right)=$ ball $_{r}\left(\phi^{n}\left(B_{\phi}\right)^{\mathrm{sa}}\right)$ for every $r>0$ and every $n=1,2, \ldots$; hence

$$
\operatorname{ball}_{r} C_{\phi}^{\mathrm{sa}}=\bigcap_{n=1}^{\infty} \operatorname{ball}_{r} \phi^{n}\left(B_{\phi}^{\mathrm{sa}}\right)=\bigcap_{n=1}^{\infty} \phi^{n}\left(\operatorname{ball}_{r} B_{\phi}^{\mathrm{sa}}\right) \subseteq M_{\infty} .
$$

The asserted inclusion $C_{\phi}=C_{\phi}^{\mathrm{sa}}+i C_{\phi}^{\mathrm{sa}} \subseteq M_{\infty}$ follows after taking the union over all positive $r$.

Assume that the asymptotic lift of $\phi: M \rightarrow M$ embeds in $M$. If $M_{\infty}$ is closed under the Jordan multiplication of $M$, then by Theorem 3.3(iii), $\phi\left\lceil_{M_{\infty}}\right.$ is an order automorphism of $M_{\infty}$, and an application of Kadison's Schwarz inequality for positive linear maps implies that $\phi$ is a Jordan automorphism of $M_{\infty}$. By Lemma 4.1, $M_{\infty} \subseteq C_{\phi}$, and we conclude that $M_{\infty}=C_{\phi}$. The converse is trivial.

It is significant that when $M_{\infty}$ is not itself closed under the Jordan product, it contains a largest $\mathrm{JW}^{*}$-algebra that is characterized as follows.

Proposition 4.3. Let $\phi: M \rightarrow M$ be a UP map whose asymptotic lift embeds in $M$. Then the weak ${ }^{*}$-closed linear span of all projections in $M_{\infty}$ is a JW*-algebra.

Proof. By Corollary 3.4, there are a $\mathrm{JW}^{*}$-algebra $B$ and an order isomorphism $\alpha$ of $B$ onto $M_{\infty}$. Viewing $\alpha$ as a UP map of $B$ into the $\mathrm{JW}^{*}$-algebra $M$, it makes sense to speak of its definite set $B_{\alpha}$. Since $B_{\alpha}$ is a JW*-subalgebra of $B$ and the restriction of $\alpha$ to $B_{\alpha}$ is a Jordan homomorphism, it follows that $\alpha\left(B_{\alpha}\right) \subseteq M_{\infty}$ is a $\mathrm{JW}^{*}$-subalgebra of $M$.

Being a $\mathrm{JW}^{*}$-algebra, $\alpha\left(B_{\alpha}\right)$ is generated by its projections. Conversely, we claim that every projection $e \in M_{\infty}$ belongs to $\alpha\left(B_{\alpha}\right)$. To see that, fix such an $e$ and choose $f \in B$ such that $\alpha(f)=e$. Then $0 \leq f \leq \mathbf{1}$ because $\alpha$ is a UP order isomorphism, so, by Kadison's Schwarz inequality,

$$
0=e-e^{2}=\alpha(f)-\alpha(f)^{2} \geq \alpha\left(f-f^{2}\right) \geq 0,
$$

hence $\alpha\left(f-f^{2}\right)=0$ and finally $f=f^{2}$. Then $\alpha\left(f^{2}\right)=\alpha(f)^{2}$ shows $f$ belongs to the definite set of $\alpha$; hence $e=\alpha(f) \in \alpha\left(B_{\alpha}\right)$.

We conclude the section by describing intrinsic conditions on a UP map which imply that its asymptotic lift is embeddable as the multiplicative core; note that the sufficient conditions of Theorem 4.5 are clearly necessary as well. 
For a linear functional $\rho$ on $M$ and a set of operators $S \subseteq M$, we write $\rho \perp S$ whenever $\rho(S)=\{0\}$. We require the following result characterizing the equality $M_{\infty}=C_{\phi}$ in terms of the action of $\phi$ on the predual of $M$ :

Lemma 4.4. Let $\phi: M \rightarrow M$ be a UP map. Then $M_{\infty}=C_{\phi}$ if and only if for every $\rho \in M_{*}$ satisfying $\rho \perp C_{\phi}$, one has

$$
\lim _{n \rightarrow \infty}\left\|\rho \circ \phi^{n}\right\|=0 .
$$

Proof. We claim first that $\rho \in M_{*}$ satisfies (4-7) if and only if $\rho \perp M_{\infty}$. Indeed, setting $M_{\infty}(r)=\cap_{n} \phi^{n}$ (ball $l_{r} M$ ) for $r>0$, and noting that the compact convex sets $\phi^{n}\left(\right.$ ball $\left._{r} M\right)$ decrease to $M_{\infty}(r)$ as $n \uparrow \infty$, we can apply [Arveson 2007, Lemma $3.5]$ to conclude that (4-7) is equivalent to the assertion

$$
\begin{aligned}
\sup \left\{|\rho(x)|: x \in M_{\infty}(r)\right\} & =\lim _{n \rightarrow \infty} \sup \left\{|\rho(x)|: x \in \phi^{n}\left(\operatorname{ball}_{r} M\right)\right\} \\
& =r \cdot \lim _{n \rightarrow \infty}\left\|\rho \circ \phi^{n}\right\|=0
\end{aligned}
$$

for every $r>0$. Noting that $M_{\infty}=\cup_{r>0} M_{\infty}(r)$, the claim follows.

The preceding paragraph, together with a standard separation theorem, shows that the assertion

$$
\rho \perp C_{\phi} \Longrightarrow \lim _{n \rightarrow \infty}\left\|\rho \circ \phi^{n}\right\|=0
$$

is equivalent to the claim ${\overline{M_{\infty}}}^{\mathrm{w} *} \subseteq C_{\phi}$. Since we generally have $C_{\phi} \subseteq M_{\infty} \subseteq{\overline{M_{\infty}}}^{\mathrm{w} *}$, (4-8) is equivalent to $C_{\phi}=M_{\infty}$.

Theorem 4.5. Let $\phi: M \rightarrow M$ be a UP map such that

(i) The positive linear map obtained by restricting $\phi$ to $C_{\phi}$ is faithful.

(ii) $\lim _{n \rightarrow \infty}\left\|\rho \circ \phi^{n}\right\|=0$ for every $\rho \in M_{*}$ satisfying $\rho \perp C_{\phi}$.

Then $M_{\infty}=C_{\phi}$, the restriction of $\phi$ to $C_{\phi}$ is a Jordan automorphism of $C_{\phi}$, and the asymptotic lift of $\phi$ is $\left(C_{\phi}, \phi\left\lceil_{C_{\phi}}, \iota\right)\right.$.

Proof. Hypothesis (i) implies that the restriction of $\phi$ to $C_{\phi}$ is a Jordan automorphism. Thus, the triple $\left(C_{\phi}, \phi \uparrow_{C_{\phi}}, \iota\right)$ is a nondegenerate reversible lift of $\phi$. To show it is the asymptotic lift, we must establish for every $\rho \in M_{*}$ the inequality

$$
\lim _{n \rightarrow \infty}\left\|\rho \circ \phi^{n}\right\| \leq \| \rho\left\lceil C_{\phi} \| .\right.
$$

For that, fix $\rho$, and, for every $n=1,2, \ldots$, choose an element $x_{n} \in M$ satisfying $\left\|x_{n}\right\|=1$ and $\left|\rho\left(\phi^{n}\left(x_{n}\right)\right)\right|=\left\|\rho \circ \phi^{n}\right\|$. We can find a subsequence $n_{1}<n_{2}<\ldots$

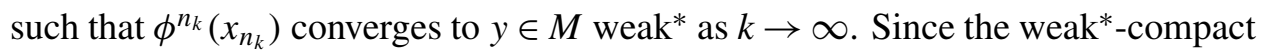
sets $\phi^{n}$ (ball $M$ ) decrease with increasing $n, y$ must belong to their intersection $\cap_{n} \phi^{n}($ ball $M)=M_{\infty}$, and of course $\|y\| \leq 1$. 
Making use of hypothesis (ii) and Lemma 4.4, we conclude that $M_{\infty}=C_{\phi}$, so that $y \in$ ball $C_{\phi}$. Hence

$$
\lim _{n \rightarrow \infty}\left\|\rho \circ \phi^{n}\right\|=\lim _{k \rightarrow \infty}\left\|\rho \circ \phi^{n_{k}}\right\|=\lim _{k \rightarrow \infty}\left|\rho\left(\phi^{n_{k}}\left(x_{n_{k}}\right)\right)\right|=|\rho(y)| \leq \| \rho\left\lceil C_{\phi} \|,\right.
$$

and the desired inequality (4-9) follows.

Remark 4.6. The conclusion of Theorem 4.5 can be significantly strengthened whenever there is a normal positive projection $E$ of $M$ onto $C_{\phi}$ - in particular, whenever $M$ is a finite von Neumann algebra. In such cases, a simple argument (which we omit) shows that the automorphism $\alpha=\phi \uparrow_{C_{\phi}}$ of $C_{\phi}$ satisfies

$$
\lim _{n \rightarrow \infty}\left\|\rho \circ \phi^{n}-\rho \circ \alpha^{n} \circ E\right\|=0 \quad \text { for } \rho \in M_{*} .
$$

\section{UP maps on finite-dimensional algebras}

In this section we show that the asymptotic lift of every UP map acting on a finitedimensional algebra $M$ embeds in $M$. We identify $M_{\infty}$ with the multiplicative core whenever there is a faithful $\phi$-invariant state, and more generally, we identify the multiplicative core when $\phi$ is faithful. We conclude with an elementary example exhibiting nontrivial asymptotic dynamics, for which $M_{\infty}$ is not closed under the Jordan product of $M$ and hence differs from the multiplicative core.

Theorem 5.1. Let $\phi: M \rightarrow M$ be a UP map on a finite-dimensional von Neumann algebra. Then $\left(M_{\infty}, \phi \uparrow_{M_{\infty}}, \iota\right)$ is the asymptotic lift of $\phi$. If, in addition, for every positive operator $x \in M$, one has

$$
\lim _{n \rightarrow \infty}\left\|\phi^{n}(x)\right\|=0 \quad \text { implies } x=0,
$$

then $M_{\infty}$ is the multiplicative core $C_{\phi}$. Condition (5-10) is satisfied whenever there is a faithful state $\rho$ of $M$ satisfying $\rho \circ \phi=\rho$.

The proof requires a known elementary result:

Lemma 5.2 [Effros and Størmer 1979]. Let $M$ be a unital $C^{*}$-algebra and let $E: M \rightarrow M$ be an idempotent UP map that is faithful, that is, $x \in M^{+}$and $E(x)=0$ implies $x=0$. Then $E(M)$ is a Jordan subalgebra of $M$.

Proof. Choose a self-adjoint element $x \in E(M)$. Then $E\left(E\left(x^{2}\right)-x^{2}\right)=E\left(x^{2}\right)-$ $E\left(x^{2}\right)=0$. By Kadison's Schwarz inequality, $x^{2}=E(x)^{2} \leq E\left(x^{2}\right)$, so that $E\left(x^{2}\right)-$ $x^{2} \geq 0$. Since $E$ is faithful, $E\left(x^{2}\right)=x^{2} \in E(M)$. This shows that $E(M)^{\text {sa }}$ is closed under the Jordan product; hence $E(M)$ is a Jordan subalgebra of $M$.

Proof of Theorem 5.1. There is a sequence $n_{1}<n_{2}<\cdots$ of positive integers such that $\phi^{n_{k}}$ converges to a unique idempotent $E$; this is a result of Kuperberg, see 
[Arveson 2004, Theorem 4.1 and what follows]. Note that $E(M)=M_{\infty}$. Indeed, for every $x \in M$,

$$
E(x)=\lim _{k} \phi^{n_{k}}(x) \in \cap_{n} \overline{\left\{\phi^{n}(x), \phi^{n+1}(x) \ldots\right\}} \subseteq \cap_{n} \phi^{n}\left(\operatorname{ball}_{\|x\|} M\right) ;
$$

hence $E(x) \subseteq M_{\infty}$. For the opposite inclusion, choose $y \in M_{\infty}$. Then there is a bounded sequence $x_{k} \in M$ such that $y=\phi^{n_{k}}\left(x_{k}\right)$ for every $k$. Let $k^{\prime}$ be a subsequence of $k$ such that $x_{k^{\prime}}$ converges to $x \in M$. Then

$$
\left\|y-\phi^{n_{k^{\prime}}}(x)\right\| \leq\left\|y-\phi^{n_{k^{\prime}}}\left(x_{k^{\prime}}\right)\right\|+\left\|\phi^{n_{k^{\prime}}}\left(x_{k^{\prime}}\right)-\phi^{n_{k^{\prime}}}(x)\right\| \leq\left\|x_{k^{\prime}}-x\right\|,
$$

and the right side tends to zero as $k^{\prime} \rightarrow \infty$.

[Arveson 2004] also shows that $\phi$ restricts to a surjective isometry on $M_{\infty}$ and that the powers of $\phi$ tend to zero on $\operatorname{ker} E$, so that

$$
\lim _{n \rightarrow \infty}\left\|\phi^{n} \circ E-\phi^{n}\right\|=0 .
$$

It follows that for every bounded linear functional $\rho$ on $M$, we have

$$
\begin{aligned}
\limsup _{n \rightarrow \infty} \mid \| \rho\left\lceil_{M_{\infty}}\|-\| \rho \circ \phi^{n} \| \mid\right. & =\limsup _{n \rightarrow \infty}\left|\left\|\rho \circ \phi^{n} \circ E\right\|-\left\|\rho \circ \phi^{n}\right\|\right| \\
& \leq \lim _{n \rightarrow \infty}\left\|\rho \circ \phi^{n} \circ E-\rho \circ \phi^{n}\right\|=0 .
\end{aligned}
$$

The latter implies that $\left(M_{\infty}, \phi\left\lceil_{\infty}, \iota\right)\right.$ is the asymptotic lift of $\phi$.

Assuming that (5-10) is satisfied, we claim that $M_{\infty}=C_{\phi}$. By Theorem 4.2, it is enough to show that $M_{\infty}$ is closed under the Jordan multiplication of $M$. Note that $E$ must be faithful. Indeed, if $x \geq 0$ and $E(x)=0$, then

$$
\lim _{k}\left\|\phi^{n_{k}}(x)\right\|=\|E(x)\|=0,
$$

and since the sequence of norms $\left\|\phi_{n}(x)\right\|$ is decreasing, (5-10) implies that $x=0$. By Lemma 5.2, $M_{\infty}$ is a Jordan subalgebra of $M$.

Finally, assuming that there is a faithful $\phi$-invariant state $\rho$, we claim that (5-10) holds. Indeed, if $x$ is a positive operator satisfying $\left\|\phi^{n}(x)\right\| \rightarrow 0$ as $n \rightarrow \infty$, then

$$
\left.|\rho(x)|=\mid \rho \circ \phi^{n}(x)\right) \mid \leq\left\|\phi^{n}(x)\right\| \rightarrow 0
$$

as $n \rightarrow \infty$. Hence $\rho(x)=0$, and $x=0$ follows because $\rho$ is faithful.

We conclude with a result which identifies the multiplicative core $C_{\phi}$ in many cases where $C_{\phi} \neq M_{\infty}$.

Corollary 5.3. Let $\phi: M \rightarrow M$ be a faithful UP map on a finite-dimensional von Neumann algebra. Then the multiplicative core of $\phi$ is the linear space spanned by the projections in $M_{\infty}$. 
Proof. Let $A$ be the linear span of all projections in $M_{\infty}$. We claim that $\phi(A)=A$. Indeed, if $e$ is a projection in $A$, then, since $\phi$ is an order automorphism of $M_{\infty}$, there is an operator $f \in M_{\infty}$ such that $0 \leq f \leq \mathbf{1}$ and $\phi(f)=e$. As in the proof of Proposition 4.3, this implies $\phi\left(f-f^{2}\right)=0$; hence $f=f^{2}$ is a projection because $\phi$ is faithful. This implies that $A \subseteq \phi(A)$ and, since $A$ is finite-dimensional, $\phi(A)=A$.

Proposition 4.3 implies that $A$ is the largest Jordan algebra in $M_{\infty}$. The multiplicative core $C_{\phi}$ is a Jordan algebra in $M_{\infty}$ by Theorem 4.2; hence $C_{\phi} \subseteq A$. Lemma 4.1 now implies that $C_{\phi}=A$.

Example 5.4. We conclude by describing an example of a UP map on the 3dimensional commutative $C^{*}$-algebra $M=\mathbb{C}^{3}$ for which $M_{\infty}$ is not closed under the ambient Jordan multiplication of $M$. While there are simpler examples with that specific property, this one exhibits nontrivial asymptotic dynamics that are not detected by the multiplicative core. Viewing the elements of $M$ as column vectors, the map $\phi$ is multiplication by the stochastic matrix

$$
\phi=\left(\begin{array}{ccc}
\frac{1}{3} & \frac{1}{3} & \frac{1}{3} \\
0 & 0 & 1 \\
0 & 1 & 0
\end{array}\right)
$$

The even and odd powers of $\phi$ are

$\phi^{2 n}=\left(\begin{array}{ccc}\frac{1}{9^{n}} & \frac{1}{2}-\frac{1}{2 \cdot 9^{n}} & \frac{1}{2}-\frac{1}{2 \cdot 9^{n}} \\ 0 & 1 & 0 \\ 0 & 0 & 1\end{array}\right) \quad$ and $\quad \phi^{2 n+1}=\left(\begin{array}{ccc}\frac{1}{3 \cdot 9^{n}} & \frac{1}{2}-\frac{1}{6 \cdot 9^{n}} & \frac{1}{2}-\frac{1}{6 \cdot 9^{n}} \\ 0 & 0 & 1 \\ 0 & 1 & 0\end{array}\right)$,

and the unique idempotent limit point of $\left\{\phi, \phi^{2}, \phi^{3}, \ldots\right\}$ is given by

$$
E=\lim _{n \rightarrow \infty} \phi^{2 n}=\left(\begin{array}{ccc}
0 & \frac{1}{2} & \frac{1}{2} \\
0 & 1 & 0 \\
0 & 0 & 1
\end{array}\right)
$$

The range of $E$ is the two-dimensional space (written as row vectors)

$$
M_{\infty}=E(M)=\left\{\left(\frac{a+b}{2}, a, b\right): a, b \in \mathbb{C}\right\} .
$$

We summarize the basic properties of this example without proof: Relative to the intrinsic (Jordan) multiplication defined by $x \circ y=E(x y)$, the space $M_{\infty}$ is isomorphic to the two-dimensional commutative $C^{*}$-algebra $\mathbb{C}^{2}$. This identification implements a conjugacy of $\phi \uparrow_{\infty}$ with the order 2 automorphism $(a, b) \mapsto(b, a)$ for $a, b \in \mathbb{C}$. The multiplicative core of $\phi$ is the one-dimensional $C^{*}$-algebra $C_{\phi}=$ $\mathbb{C} \cdot 1$. 
Remark 5.5 (Invariant states of $\phi$ ). The above UP map $\phi: \mathbb{C}^{3} \rightarrow \mathbb{C}^{3}$ has a unique invariant state, namely

$$
\rho(a, b, c)=\frac{b+c}{2} \quad \text { for }(a, b, c) \in \mathbb{C}^{3} .
$$

This state is not faithful of course, so there is no conflict with Theorem 5.1.

\section{References}

[Arveson 2004] W. Arveson, "Asymptotic stability. I. Completely positive maps", Internat. J. Math. 15:3 (2004), 289-312. MR 2005g:46124 Zbl 1065.46047

[Arveson 2007] W. Arveson, "The asymptotic lift of a completely positive map", J. Funct. Anal. 248:1 (2007), 202-224. MR 2329688 Zbl 05171415

[Effros and Størmer 1979] E. G. Effros and E. Størmer, "Positive projections and Jordan structure in operator algebras”, Math. Scand. 45:1 (1979), 127-138. MR 82e:46076 Zbl 0455.46059

[Russo and Dye 1966] B. Russo and H. A. Dye, "A note on unitary operators in $C^{*}$-algebras", Duke Math. J. 33 (1966), 413-416. MR 33 \#1750 Zbl 0171.11503

[Størmer 2007] E. Størmer, "Multiplicative properties of positive maps”, Math. Scand. 100:1 (2007), 184-192. MR 2331197

Received November 15, 2006.

WILLIAM ARVESON

DEPARTMENT OF MATHEMATICS

UNIVERSITY OF CALIFORNIA

BERKELEY, CA 94720

UNITED STATES

arveson@math.berkeley.edu

http://math.berkeley.edu/ arveson/

ERLING STøRMER

UNIVERSITY OF OSLO

P.O. BOX 1053

0316 OSLO

NORWAY

erlings@math.uio.no 